\title{
Analyzing the most frequent disease loci in targeted patient categories optimizes disease gene identification and test accuracy worldwide
}

Roger V Lebo ${ }^{1,2^{*}}$ and Vijay S Tonk ${ }^{3,4}$

\begin{abstract}
Background: Our genomewide studies support targeted testing the most frequent genetic diseases by patient category: (1) pregnant patients, (2) at-risk conceptuses, (3) affected children, and (4) abnormal adults. This approach not only identifies most reported disease causing sequences accurately, but also minimizes incorrectly identified additional disease causing loci.
\end{abstract}

Methods: Diseases were grouped in descending order of occurrence from four data sets: (1) GeneTests 534 listed population prevalences, (2) 4129 high risk prenatal karyotypes, (3) 1265 affected patient microarrays, and (4) reanalysis of 25,452 asymptomatic patient results screened prenatally for 108 genetic diseases. These most frequent diseases are categorized by transmission: (A) autosomal recessive, (B) X-linked, (C) autosomal dominant, (D) microscopic chromosome rearrangements, (E) submicroscopic copy number changes, and (F) frequent ethnic diseases.

Results: Among affected and carrier patients worldwide, most reported mutant genes would be identified correctly according to one of four patient categories from at-risk couples with $<64$ tested genes to affected adults with 314 tested loci. Three clinically reported patient series confirmed this approach. First, only 54 targeted chromosomal sites would have detected all 938 microscopically visible unbalanced karyotypes among 4129 karyotyped POC, CVS, and amniocentesis samples. Second, 37 of 48 reported aneuploid regions were found among our 1265 clinical microarrays confirming the locations of 8 schizophrenia loci and 20 aneuploidies altering intellectual ability, while also identifying 9 of the most frequent deletion syndromes. Third, testing 15 frequent genes would have identified 124 couples with a 1 in 4 risk of a fetus with a recessive disease compared to the 127 couples identified by testing all 108 genes, while testing all mutations in 15 genes could have identified more couples.

Conclusion: Testing the most frequent disease causing abnormalities in 1 of 8 reported disease loci [ 1 of 84 total genes] will identify $\sim 7$ of 8 reported abnormal Caucasian newborn genotypes. This would eliminate $\sim 8$ to 10 of $\sim 10$ Caucasian newborn gene sequences selected as abnormal that are actually normal variants identified when testing all $\sim 2500$ diseases looking for the remaining 1 of 8 disease causing genes. This approach enables more accurate testing within available laboratory and reimbursement resources.

Keywords: Most frequent diseases, Targeted patient categories, Disease identification, Test accuracy

\footnotetext{
* Correspondence: rlebo@chmca.org

'Department of Pathology and Laboratory Medicine, Akron Children's

Hospital, One Perkins Square, Akron, OH 44308-1062, USA

${ }^{2}$ Northeast Ohio Medical University, Rootstown, OH, USA

Full list of author information is available at the end of the article
} 


\section{Background}

Targeted testing the most frequent listed disease causing sites comprising $<0.3 \%$ to $1.5 \%$ [This study] of the $\sim 22,000$ individual gene locations causing $\sim 2500$ total reported diseases [1] will minimize the number of incorrectly identified abnormal gene sequences by excluding nearly all normally variant sequences. By minimizing interpretation time and confirmation of abnormal results, this will enableready identification of most disease causing genomic sequences to expedite patient testing.

All laboratory tests have limitations. Because positive genetic test results may provide the basis for clinical decisions on both patients and their relatives for many years, clinical laboratories continually strive to offer optimal tests that maintain the highest possible reported accuracy. Genomewide analysis is limited by genome complexity so that accurate interpretation of all test results can be challenging. For instance, genomewide microarray analysis of $1,800,000$ sites for disease causing rearrangements also identifies 906,600 polymorphisms and 5,677 copy number variants [2] among the $\sim 2,370,000$ total listed copy number variants [3]. Genomewide next generation sequencing continues to identify reported genetic mutations with ever more accurate, rapid, less expensive platforms that can analyze a few selected genes up to all $\sim 22,000$ genes in the $6,000,000,000$ basepair diploid genome. Yet the more sites tested, the more normal sites are identified as abnormal by available data analysis. Currently "the rate limiting factor in clinical genomewide testing is the numerous variant gene sequences that multiply the cost of interpreting the raw sequence about 10-fold" [4].

Dr. James Watson, a healthy senior scientist was among the first three individuals to have his entire genome sequenced [5,6]. Three computer programs found Dr. Watson's genome includes (1) two homozygous variants in genes previously reported to cause Usher Syndrome $1 \mathrm{~b}$ and Cockayne syndrome, both early childhood diseases [7], and (2) a breast cancer gene sequence originally interpreted to reflect a major mutation among his $>80$ described mutated alleles. Subsequent reinterpretation of his known breast cancer gene variant avoided further clinical intervention [6]. None of these three diseases are among the most frequent selected for routine genomewide testing (Additional file 1: Table S1, Additional file 2: Table S2, Additional file 3: Table S3, Table 1).

Carrier screening for cystic fibrosis has been applied to millions of patients and their at-risk partners according to this current standard-of-care DNA test $[8,9]$ reported as either positive or with a residual negative test risk $[10,11]$. Newborn Screening Programs in every state test for selected abnormal metabolite concentrations by mass spec while some states also test newborns for cystic fibrosis mutations to optimize follow up care. [Additional file 1: Table S1A, Column 2, NB tested in Ohio] Frequent disease gene mutation screening selected for Ashkenazi patients by ACMG [12] and completed on tens of thousands of Ashkenazi patients from New York to Tel Aviv [13] is attributed with substantially reducing the frequency of affected newborns with these ethnic diseases.

Platforms that sequence the exome and those that quantify the copy number of targeted sites [14-16] together enable genomewide analysis to identify (1) single nucleotide substitutions, (2) gene deletions revealed by FISH, and (3) unbalanced chromosome region copy number abnormalities identified by karyotypes and precisely delineated by microarrays. Standard karyotyping is still the preferred method for detecting balanced and complex rearrangements, as microarray and sequencing methods are being validated to detect balanced abnormalities ([17], Results). Additional modifications to DNA analysis platforms have enabled sequencing single nucleotide mutations by microarrays [18] while other computer programs count and list the number of copies of each selected sequenced locus to quantify abnormal genomic sites c.f. [19].

Targeting genomewide screening the most frequent genetic diseases affecting the largest proportion of patients worldwide with rapid analysis platforms will enable unambiguously identifying more abnormal genotypes in at-risk couples and affected conceptuses and patients with fewer confounding results [Tables 1 and 2]. Testing products of conception and fetuses for the most frequent chromosome aneuploidies identifies the largest proportion of abnormal conceptuses [Additional file 2: Table S2, Col 2,3,4]. Testing abnormal infants and children identifies a substantial proportion of patients with altered intellectual development caused by a frequently deleted or duplicated submicroscopic chromosome region (Additional file 3: Table S3). Simultaneously testing for the other most frequent dominant and recessive single gene disorders including those in appropriate ethnic populations [Additional file 1: Table S1F] can provide accurate results to large patient populations within medical, laboratory, and reimbursement resources [Table 1].

\section{Methods}

\section{Design of the study}

This study tested the hypothesis that analyzing the most frequent genetic diseases selected from all reported diseases would identify the largest proportion of disease causing mutations to unambiguously define each positive testing patient's genetic abnormality with very few incorrect test results. For instance, when testing 100,000 patients for the frequent autosomal recessive cystic fibrosis mutations with $99.9 \%$ test accuracy per gene, a positive 
Table 1 Total population frequencies (Additional file 1: Table S1A, B, C, D3, E right) by selected tested patient categories (Additional file 1: Table S1D1, D2, E2)

\begin{tabular}{|c|c|c|c|c|}
\hline \multirow{2}{*}{$\begin{array}{l}\text { Disease categories } \\
\text { Tested }\end{array}$} & \multicolumn{2}{|c|}{ Caucasian } & \multicolumn{2}{|c|}{ Worldwide+ } \\
\hline & (a) Affected & (b) Heterozygote & (a') Affected & (b') Heterozygote \\
\hline 1. COUPLES, Asymptomatic, at risk & & $\sim 1 / 132$ & & $\sim 1 / 174$ \\
\hline$(1 A 2 b+1 B 2 b)($ ASYMPTOMATIC, & & ( .76\%) & & ( .58\%) \\
\hline 2. FETUSES, Abnormal & $\sim 1 / 11.0$ & & $(1 / 11.1)$ & \\
\hline$(1 A 1 a+1 B 1 a+2 A 1+3 A)$ & ( 9.2\%) & & ( 9.0\%) & \\
\hline 3. NEWBORNS, Affected & $\sim 1 / 52$ & & $\sim 1 / 54$ & \\
\hline$(1 A 1 a+1 B 1 a+1 C a+2 c+3 A)$ & $\sim 1.91 \%$ & & $\sim 1.86 \%$ & \\
\hline 4. ADULTS(a), Affected & $\sim 1 / 37$ & & & $\sim 1 / 43$ \\
\hline$(1 A 1 a, 1 B 1 a+1 C a+1 D a+2 A+3 A)$ & $\sim 2.71 \%$ & & $\sim 2.33 \%$ & \\
\hline
\end{tabular}

Testing for Abnormal Genotypes in Asymptomatic Adults and Symptomatic Fetuses, Newborns, Children, and Adults.

Frequencies for each disease category are listed in Additional file 1: Table S1 according to the frequency in the general population. Clinically affected patients tested for any age-appropriate category carry substantially greater frequencies of affected genotypes (Additional file 2: Table S2 and Additional file 3: Table S3). Age appropriate tests are anticipated to optimally identify specific diseases in affected patients according to patient category (Table 4).

carrier test would include 3445 correct answers and 103 incorrect answers (Table 3, top; Ref. [20]). In contrast, when testing the rare fumarase deficiency gene locus with the same test accuracy, a positive carrier test would not only identify 26 carriers correctly but also 100 noncarriers as carriers [Table 3 , bottom]. Thus, a minimal frequency of $\sim 1$ in 100,000 affected individuals for each listed abnormality was arbitrarily selected in each population analyzed to minimize incorrect test results while maximizing the number of genetic abnormalities identified. Available patient studies with the largest summarized experience [1] were selected to compile the abnormal gene frequencies in populations.

\section{Study setting}

The diseases listed in descending order of frequency were identified from: (1) GeneTests 534 listed disease prevalences affecting at least 1 in 100,000 people among

Table 2 Most frequent disease gene categories tested in patients

\begin{tabular}{|c|c|c|c|c|}
\hline & $\begin{array}{l}\text { Autosomal/ } \\
\text { X-Linked } \\
\text { recessive }\end{array}$ & $\begin{array}{l}\text { Chromosome/ } \\
\text { Gene R }\end{array}$ & $\begin{array}{l}\text { Autosomal } \\
\text { dominant/ } \\
\text { Aneuploidy }\end{array}$ & $\begin{array}{l}\text { Late } \\
\text { onset } \\
\text { disease }\end{array}$ \\
\hline $\begin{array}{l}\text { 1. Reproducing or } \\
\text { selecting partner }\end{array}$ & $x$ & & & \\
\hline $\begin{array}{l}\text { 2. At-risk conceptus } \\
\text { or fetus }\end{array}$ & $x$ & $x$ & & \\
\hline $\begin{array}{l}\text { 3. Affected newborn } \\
\text { or minor }\end{array}$ & $x$ & $x$ & $x$ & \\
\hline 4. Affected adult & $x$ & $x$ & $x$ & $x$ \\
\hline
\end{tabular}

Legend: Carrier screening includes asymptomatic patients selecting partners, planning to conceive, or pregnant, and partners of identified carriers. Prenatal testing includes products of conception and at-risk fetuses. Symptomatic newborns and minors can be tested for autosomal dominant disease loci to determine the cause of their abnormal phenotype. Adults could be tested for selected late-onset disease genes and males for Y-linked infertility. the $>2500$ listed diseases, (2) our 4129 reported products of conception and prenatal karyotypes in Ohio, (3) our 1265 reported patient microarray results in Texas, and (4) reanalyzed results of 25,452 prenatally screened women and their at risk partners tested for 108 disease genes [16].

\section{Type of participants and materials}

The abnormal clinically reported prenatal karyotypes were derived from 1,449 products of conception (POCs), 82 chorionic villus samples, and 2598 amniocenteses completed at Akron Children's Hospital from 2002 to 2013 (4,129 total cases). For comparison, we added the substantially lower frequencies of our previously published abnormal results on 25,222 amniocenteses and 5,134 chorionic villus samplings with a substantially larger proportion of patients of advanced maternal age among those cases with abnormal ultrasounds completed by 1992 [21].

The 121 (9.6\%) clinically reported abnormal microarray results from 1265 Texas' (T) patients were submitted for phenotypic abnormalities unrelated to oncology. These 40 different identified submicroscopic deletions and duplications each spanning about $\sim 2,000,000$ basepairs were reported with references in the Agilent and/ or BlueGnome databases (Additional file 3: Table S3A, Ref. $[22,23])$. The positive results at each genomic locus were listed initially according to the relative frequency of each abnormal site observed [Additional file 3: Table S3A, Col 4, Left]. The frequencies reported in another developmentally delayed population of 15,749 cases and 10,118 controls [24] were added on the right side of the affected column for comparison [Additional file 3: Table S3A, Col.4, Right, (K)]. Available reported abnormal copy number frequencies in the general population were added in the next column for comparison [Additional file 3: Table S3A, Col 5]. 
Table 3 Carrier test accuracies for frequent and rare autosomal recessive diseases [20]

\begin{tabular}{|c|c|c|c|c|}
\hline Disease & Disease frequency & $98 \%$ Accurate $* 100,000$ tested & $99.9 \%$ Accurate ${ }^{* *} 100,000$ tested & Carrier frequency \\
\hline \multirow[t]{3}{*}{ Cystic Fibrosis } & $\sim 1 / 3364$ & $\sim 3279$ Correct & $\sim 3445$ Correct & $\sim 1 / 29$ \\
\hline & & 2067 Incorrect & 103 Incorrect & \\
\hline & & $(2000+67=2067)$ & $(100+3.3=103.3)$ & \\
\hline \multirow[t]{3}{*}{ PKU } & $\sim 1 / 10,000$ & 1960 Correct & 1998 Correct & $\sim 1 / 50$ \\
\hline & & 2040 Incorrect & 102 Incorrect & \\
\hline & & $(200+40=2040)$ & $(100+2=102)$ & \\
\hline Arylsulfatase A & $\sim 1 / 100,000$ & $\sim 619$ Correct & 632 Correct & $\sim 1 / 158$ \\
\hline \multirow[t]{2}{*}{ Deficiency } & & 2012 Incorrect & 101 Incorrect & \\
\hline & & $(2000+12.4=2012.4)$ & $(100+.6=100.6)$ & \\
\hline Fumarese & $\sim 1 / 60,000,000$ & $\sim 25$ Correct & $\sim 26$ Correct & $\sim 1 / 3873$ \\
\hline \multirow[t]{2}{*}{ Deficiency } & & 2000 Incorrect & $\sim 100$ Incorrect & \\
\hline & & $(2000+.5=2000.5)$ & $(100+.026=100.026)$ & \\
\hline
\end{tabular}

${ }^{*}$ A $96 \%$ to $98 \%$ accurate cystic fibrosis result frequency was initially reported by CAP certified testing laboratories.

**A $99.5 \%$ accurate result frequency was estimated by one commercial microarray manufacturer.

These data illustrate the prudence of testing maternal and fetal samples together.

LEGEND: One first reason for targeting the most frequent genetic diseases is illustrated by the calculated differences between correct and incorrect test results for diseases with different frequencies given the same test accuracies. For instance, considerably higher test accuracies are observed when calculated for screening of the more frequent autosomal recessive diseases in unselected asymptomatic carriers. The proportion of incorrectly detected carriers increases substantially for rare autosomal recessive diseases like Fumarase deficiency.

Clinical test accuracy is optimized during laboratory validation according to College of American Pathology guidelines. An illustrative $98 \%$ test accuracy has been arbitrarily selected for comparison of a Standard of Care test based upon the $96 \%$ to $98 \%$ accurate cystic fibrosis results reported by CAP certified clinical laboratories initially screening for the 23 most common cystic fibrosis mutations. Given a test accuracy of $98 \%$ for cystic fibrosis would identify 3279 cystic fibrosis carriers correctly and $\sim 67$ carriers and $\sim 2000$ noncarriers incorrectly among 100,000 people. The same test accuracy applied to the rare autosomal recessive fumarase deficiency with a frequency of $\sim 1$ in 60,000,000 would identify 25 of 26 fumarase deficiency carriers correctly but also identify 1 carrier and 2000 noncarriers incorrectly.

DNA sequencing platforms themselves are anticipated to be substantially more accurate, while entire test accuracy is also modified by sample collection, laboratory manipulation, and reporting. An arbitrarily selected $99.9 \%$ accurate test would decrease the incorrectly identified noncarriers for each genetic disease from $~ 2000$ to $\sim 100$ among 100,000 patients tested. At the same time the number of correctly detected cystic fibrosis carriers would increase by 66 to 3445 . In contrast, the 26 true carriers of the rare fumarase deficiency with a frequency of 1 in 60,000,000 would be identified correctly among the 100 incorrectly identified carriers. Compare these to the calculated $99.9 \%$ accurate test results for autosomal recessive Arylsulfatase A deficiency with an affected frequency of 1 in 100,000 that would identify 632 carriers correctly along with 1 carrier and 100 noncarriers incorrectly.

The $\sim 50$-fold enriched frequency of most frequent deletions found among all patients submitted for microarray analysis (Additional file 3: Table S3B, top) illustrates the principle that testing clinically suspicious phenotypes substantially enhances the affected patient frequency among tested samples. Prior screening test results like hemoglobin electrophoresis for sickle cell anemia and the hemoglobinopathies will further enrich for abnormal patient samples submitted for DNA analysis.

These data were segregated further according to diseases with published population frequencies at the top in the order of chromosomal location for ready comparison [Additional file 3: Table S3B, top]. The remaining list was further segregated and ordered according to the chromosomal location of frequent deletions and duplications in patients with altered intellectual development [Additional file 3: Table S3B, middle], followed by patients with other clinical abnormalities [Additional file 3: Table S3B, bottom].

\section{Analysis}

All available 534 frequencies reported in the GeneReviews chapters under Prevalence were collected in 2011 and updated when a significant change was noted. This selected disease list is further organized by transmission category: (A) autosomal recessive, (B) X-linked, and (C) autosomal dominant [Additional file 1: Table S1A,B,C]. Then Y-linked and mitochondrial diseases were listed just prior to the frequent diseases in specific populations [Additional file 1: Table S1D,E,F].
Then the abnormal post conception karyotype categories were listed in order of frequency in products of conception [Additional file 2: Table S2A, Col 2]. These included (1) 1449 Products of Conception analyzed from 2002 to 2013 in Ohio, (2) the first 5,134 CVS samples tested in San Francisco [21] next to the 82 high risk samples karyotyped in Akron since 2002, (3) the first 21,288 amniocenteses karyotyped in San Francisco [21] next to the 2,598 karyotyped in Akron since 2002, and (3) 54,749 newborns karyotyped in Seattle [25].

General population frequencies used in these calculations include $50 \%$ of abnormal karyotyped products of conception [POC] in Ohio by 2013 and $0.6 \%$ of abnormal karyotypes in newborns in Seattle by 1986 (Additional file 2: Table S2B, Col 2,5). In contrast, for prenatally sampled higher risk fetuses, abnormal karyotypes reported in chorionic villous samplings [CVS] and amniocenteses completed more recently in Ohio were compared to those completed by 1992 in San Francisco (Ref. [21]; Additional file 2: Table S2A,B, Columns 3,4). 
The most frequent 48 submicroscopic aneuploid loci [Additional file 3: Table S3A] and their observed and reported frequencies were compiled from several sources: (1) The clinically reported 40 submicroscopic deletion and duplication sites each spanning $>400,000$ basepairs found in 1265 patients [Additional file 3: Table S3A, Texas (T); this manuscript], (2) thirteen (13) of the sixteen (16) deletions with estimated general population frequencies of at least 1 in 100,000 selected from GeneTests [Additional file 3: Table S3B, Top], (3) an additional 23 chromosome regions reported to result in altered neurocognitive development when deleted and occasionally when duplicated [Additional file 3: Table S3B, Middle], and (4) the additional 12 clinically reported abnormalities identified among our 1265 constitutional microarrays [Additional file 3: Table S3B, Bottom]. The first 13 are primarily syndromic deletions that frequently result in developmental delay [Additional file 3: Table S3B, Top]. The next group is reported to result in intellectual delay with or without other abnormalities. When testing for all these 48 abnormalities in 1265 patients referred for microarrays, $10 \%$ were reported positive $(\mathrm{T}): \sim 5 \%$ in the first 13 loci [Additional file 3: Table S3B, Top] and $\sim 5 \%$ in the remaining 35 loci [Additional file 3: Table S3B, Middle, Bottom].

\section{Results}

\section{Selection of frequent diseases}

The most frequent listed disease locations comprise the largest proportion of testable disease causing mutations worldwide. Estimated disease frequencies found in all tested categories were derived from our calculations based upon reported patient and general population data [Additional file 1: Table S1, Additional file 2: Table S2 and Additional file 3: Table S3]. The most frequent disease alleles for cystic fibrosis and the hemoglobinopathies are reported to have been selected by heterozygous advantage [26-28]. In contrast, the other most frequent autosomal recessive genetic disease genes have many unique alleles but none were reported to have sufficiently frequent mutations that comprise a major proportion of all mutations (Additional file 1: Table S1A; Ref. [29]). Thus determining whether a variant gene sequence is normal at a frequent disease gene site should include a comparison to all confirmed mutations.

Other frequent diseases in ethnic populations result from a limited founder pool with offspring who regularly select a partner from among the offspring of all the founders [Additional file 1: Table S1F]. These ethnic populations can be tested effectively by targeting the few most frequent mutations in the founders' rare disease alleles. Initially a population disease frequency may be overestimated when sampled from a region with a higher carrier frequency. Disease frequencies in founder populations can also skew panethnic population frequencies when offspring migrate together to specific geographic regions like in the United States where more centrally located populations can more often trace their ancestors to citizens who arrived earlier in the nation's history.

The most extensive available disease frequency estimates with the broadest geographical distributions were found on the GeneTests website in the GeneReviews chapters. This study considered all the $>2500$ listed disease genes [1] for inclusion in at-risk and affected patient test categories. The disease list selected was further organized by transmission category: (A) autosomal recessive, (B) X-linked, (C) autosomal dominant, (D) Ylinked, (E) mitochondrial, and (F) frequent diseases in specific populations [Additional file 1: Table S1A-F, Tables 1 and 4]. From the 534 listed and updated GeneTests frequencies, 125 diseases were selected that are each reported to affect at least 1 in 100,000 Caucasian individuals [Additional file 1: Table S1A,B,C,D] including 122 diseases reported to affect at least 1 in 100,000 people worldwide [Additional file 1: Table S1A,B,C,D, excluding $\# 1,2,38]$ and 55 disease genes frequent in specific populations [Additional file 1: Table S1F].

Additional estimates of disease gene frequencies have been derived from our tested at-risk and abnormal patient samples [Additional file 2: Table S2, Col 2.3.4; Additional file 3: Table S3]. Initially the abnormal karyotype categories are listed according to decreasing frequency in clinically reported products of conception [Additional file 2: Table S2A]. These karyotype results were then reorganized according to estimated severity to facilitate comparison of the remaining viable karyotypic abnormalities as gestation progresses [Additional file 2: Table S2B]. Calculated general population disease frequencies include $50 \%$ of abnormal karyotyped products of conception [POC] and $0.64 \%$ of abnormal karyotypes in newborns [Additional file 2: Table S2B, Col 2,5, Bottom]. In contrast, for prenatally sampled fetuses tested by chorionic villus sampling [CVS], abnormal karyotypes were reported in 3.1\% completed by 1992 in San Francisco [21] compared to $33 \%$ completed more recently in Ohio (Additional file 2: Table S2B, Col 3]). Note also the increased number of abnormal chromosome categories in the more recent sampled amniocenteses [Additional file 2: Table S2B, Col 4, Right, underlined).

Then the most frequent 40 aneuploid locations spanning $>400,000$ basepairs characterized by our microarrays were organized according to the frequencies of each abnormality among our 1265 tested patients [Additional file 3: Table S3A]. These frequencies among Texas' patients $(\mathrm{T})$ were subsequently reorganized according to chromosome location within 3 categories to facilitate comparison of (1) the 13 confirmed gene loci with estimated general population frequencies of at 
Table 4 Summary of disease frequencies in total population

\begin{tabular}{|c|c|c|c|c|}
\hline \multirow[t]{2}{*}{ Disease category } & \multicolumn{2}{|l|}{ Caucasian } & \multicolumn{2}{|l|}{ Worldwide(f) } \\
\hline & (a)Affected & (b) Heterozygote & (a') Affected & (b') Heterozygote \\
\hline A1. Autosomal & $\sim 1 / 668(\mathrm{a})$ & $\sim 2 / 5$ & $\sim 1 / 967$ & $\sim 1 / 3$ \\
\hline Recessive & $\sim(0.15 \%)$ & $(\sim 40 \%)$ & $(0.10 \%)$ & $(\sim 34 \%)$ \\
\hline \multirow[t]{2}{*}{ A2. Couples } & & $\sim 1 / 174$ & & $\sim 1 / 255$ \\
\hline & & $(\sim 0.58 \%)$ & & ( 0.39\%) \\
\hline \multicolumn{5}{|l|}{ A3. Late Onset } \\
\hline Parkinson (ww) & $\sim 1 / 120$ & $\sim 1 / 3.9^{*}$ & $\sim 1 / 200$ & $\sim 1 / 7$ \\
\hline Hemochromatosis (cau) (0.83\%) & $(\sim 26 \%)$ & $(0.5 \%)$ & $(\sim 14 \%)$ & \\
\hline \multirow[t]{2}{*}{ B1. X-Linked } & $\sim 1 / 1065$ & $\sim 1 / 546$ & $\sim 1 / 1065$ & $\sim 1 / 546$ \\
\hline & $\sim(0.094 \%)$ & $(\sim .18 \%)$ & $\sim(0.094 \%)$ & $(\sim .18 \%)$ \\
\hline B2. Couples & & $\sim 1 / 546$ & & $\sim 1 / 546$ \\
\hline (Recessive) & & & $(\sim .18 \%)$ & $(\sim .18 \%)$ \\
\hline C. Autosomal & $\sim 1 / 123$ & $\sim 1 / 123$ & $\sim 1 / 123$ & $\sim 1 / 123$ \\
\hline Dominant & $\sim(0.81 \%)$ & ( .81\%) & $\sim(0.81 \%)$ & $(\sim .81 \%)$ \\
\hline D1. Abn POC & $\sim 1 / 2$ & $\sim 1 / 2$ & $\sim 1 / 2$ & $\sim 1 / 2$ \\
\hline Karyotype & $\sim(50 \%)$ & $\sim(50 \%)$ & $\sim(50 \%)$ & $\sim(50 \%)$ \\
\hline Quantification (54) & $\sim(47.7 \%)$ & $\sim(47.7 \%)$ & $\sim(47.7 \%)$ & $\sim(47.7 \%)$ \\
\hline D2. Current Abn Amnio & $\sim 1 / 13.8$ & $\sim 1 / 13.8$ & $\sim 1 / 13.8$ & $\sim 1 / 13.8$ \\
\hline Karyotype & $\sim(7.2 \%)$ & $\sim(7.2 \%)$ & $\sim(7.2 \%)$ & $\sim(7.2 \%)$ \\
\hline Quantification (54) & $\sim(6.1 \%)$ & $\sim(6.1 \%)$ & $\sim(6.1 \%)$ & $\sim(6.1 \%)$ \\
\hline D3. Abnormal Newborn & $\sim 1 / 156$ & $\sim 1 / 156$ & $\sim 1 / 156$ & $\sim 1 / 156$ \\
\hline Karyotype & $\sim(0.64 \%)$ & $\sim(0.64 \%)$ & $\sim(0.64 \%)$ & $\sim(0.64 \%)$ \\
\hline Quantification (54) & $\sim(0.59 \%)$ & $\sim(0.59 \%)$ & $\sim(0.59 \%)$ & $\sim(0.59 \%)$ \\
\hline E. Common & $\sim 1 / 1097 X 2(e)$ & 1/1097X 2(e) & $\sim 1 / 1097 X 2(e)$ & $\sim 1 / 1097 X 2(e)$ \\
\hline Deletions & $\sim(0.18 \%)$ & $(\sim .18 \%)$ & $\sim(0.18 \%)$ & $(\sim .18 \%)$ \\
\hline F. Y-linked & $\sim 1 / 12,500$ & & $\sim 1 / 12,500$ & \\
\hline Hemizygote & $\sim(0.008 \%)$ & & $\sim(0.008 \%)$ & \\
\hline
\end{tabular}

(a) Without late onset hemochromatosis and Parkinson, with cystic fibrosis and a-1-antitrypsin in Caucasians.

(b) E1. First 5 abnormal karyotypes listed in Additional file 1: Table S1E detect 1/329 of 1/184 [.31\% of .54\%]. Other abnormalities may not be detected with targeted platform.

(c) E2. Other karyotypic abnormalities that may not be identified by a targeted platform. Lebo et al., 2002 , lists additional 30 chromosome regions that would identify $\sim 97 \%$ of all abnormalities if tested for copy number. Platforms with SNPs will identify copy number changes in any region in which these are found. (d) Adult estimate excludes trisomy 13 and trisomy 18 from category (b) above.

WW $=$ worldwide; Cau $=$ Caucasian.

${ }^{*}$ Compare to Cystic fibrosis: $\left.[1 / 29]^{2} \times[1 / 4]=1 / 3300\right]$. Includes hemochromatosis.

(e) The frequency of the common deletions listed at the top of Additional file 1: Table S1E comprised $5 \%$ of the abnormalities identified by microarrays while the remaining 35 loci at the bottom comprised the other $5 \%$. Thus the first population frequency was multipled by 2 to estimate the total frequency.

(f) Without common regional diseases.

least 1 in 100,000, (Additional file 3: Table S3B, Top, Right Column, Ref. [1]) (2) the additional reported chromosome regions that result in altered neurocognitive development with or without other abnormalities ((K); Additional file 3: Table S3B, Col 4; Ref. [24]), and (3) the remaining 12 abnormal clinically reported loci. Nearly all frequent deletions listed at the top of Additional file 3: Table S3B also result in altered developmental delay. When testing for all these abnormalities, 10\% of patients were reported positive for gene deletion or duplication in this Texas' cohort.
The frequencies of six disease categories with available general population frequencies [Additional file 1: Table S1A,B,C; Additional file 2: Table S2, Newborn; Additional file 3: Table S3B,Top] were calculated and graphed for ready interpretation [Figure $1 \mathrm{~A}, \mathrm{~B}, \mathrm{C}$ ]. Note that the largest proportion of reported disease frequencies is found in the first subcategory $[1 / 1$ to $1 / 25,000]$. These proportions diminish rapidly as the affected patient frequency decreases in increments to $[<1 / 75,000$ to $1 / 100,000]$. This consistent trend can be applied to optimally select disease inclusion frequency as ongoing test experience accumulates. These 

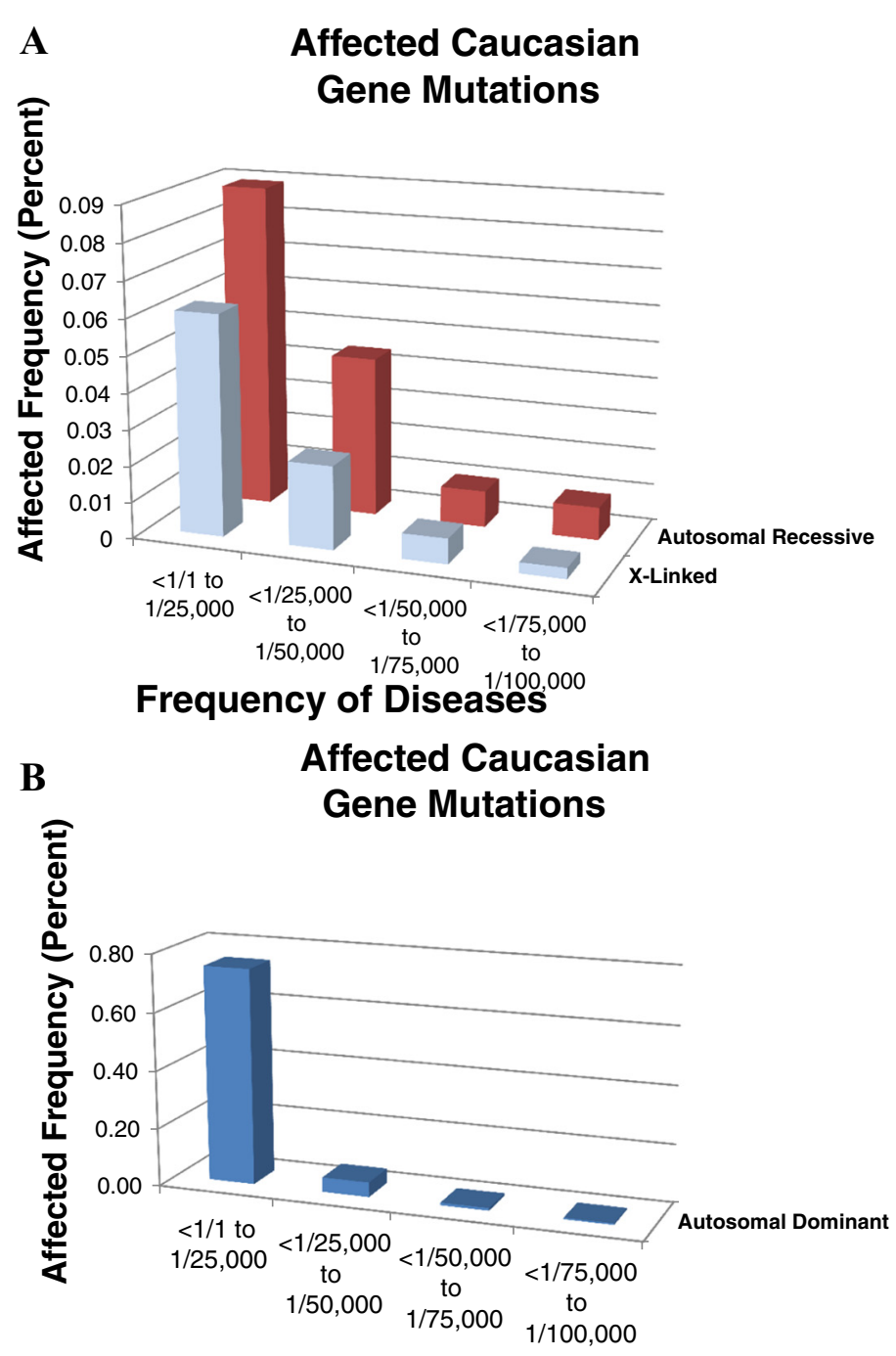

Frequency of Diseases

C

Affected Caucasian Chromosomal Abnormalities

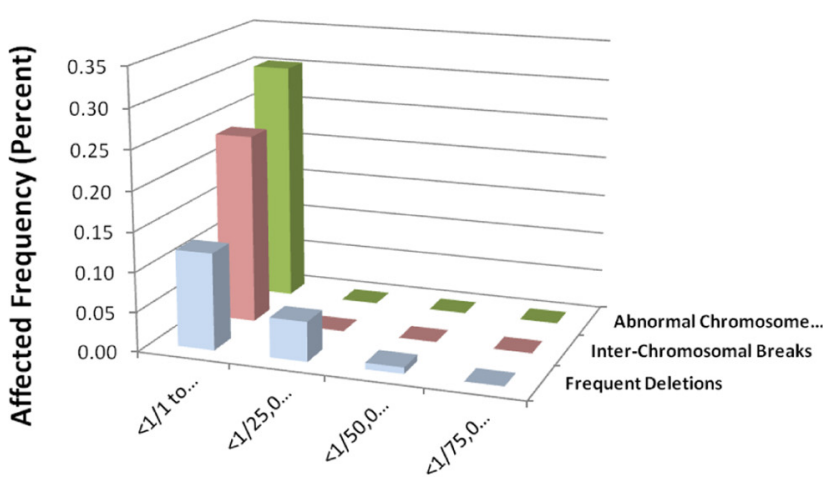

Frequency of Diseases 
(See figure on previous page.)

Figure 1 Affected disease frequencies in four disease categories in caucasians $(A, B, C)$. The individual contributions of four disease frequency categories were graphed according to affected total frequencies (percent) for 6 disease categories of surviving patients in increments of 1 in 25,000. Note the frequencies of the first three categories were graphed with a frequency up to .09\% for autosomal recessive, (Additional file 3: Table S3C), three categories were graphed on different scales with a frequency up to.30\% for whole chromosome aneuploidy (Additional file 3: Table S3E), and autosomal dominant with a frequency up to .75\%. 1. Among all the diseases with a frequency of at least 1 in $100,000,86 \%$ of at-risk couples for an affected fetus with an autosomal recessive disease would be identified by testing only diseases with a frequency up to 1 in 50,000; $2.91 \%$ of at-risk couples for an affected fetus with an X-linked disease would be identified by testing only diseases with a frequency up to 1 in 50,000; 3. 92\% of the patients affected with an auatosomal dominant would be identified by testing only diseases with a frequency up to 1 in 25,000; and 98\% with frequencies up to 1 in 50,000; and 4. All frequent duplications and chromosome abnormalities listed have frequencies exceeding 1 in 25,000. Given that most of these autosomal recessive disease genes have $\sim 50$ unique mutations with no particularly common mutations, [29], decreasing initial screening to diseases with at least 1 in 50,000 will not only substantially reduce the workload but will miss $<1$ patient per disease category in 2.5 years by a laboratory randomly screening 5,000 normal patients per year. These thresholds may need to be revised because the abnormal genomic frequencies of affected patients would be substantially greater.

graphs demonstrate that any platform that tests the most frequent disease genes according to physician selected or peer reviewed disease categories will expedite reporting the largest proportion of clearly positive and negative test results.

Altogether the first 227 [125+54+48; Additional file 1: Table S1, Col.1; Additional file 2: Table S2, Table 5; Additional file 3: Table S3A] most frequent Caucasian diseases at 263 loci $[227+36$; See Additional file 1: Table S1A,B,C,D, Column 4] result from mutations involving $\sim 1.2 \%$ of the $\sim 22,000$ human genes $[263 / 22,000=$ 1.19\%]. This total is 224 diseases for worldwide patients $[122+54+48]$. These values facilitated deriving the frequencies of at-risk asymptomatic couples carrying autosomal recessive [Table 4.A.2] and $\mathrm{X}$-linked recessive diseases (Table 4.B.2) along with the dominant and Ylinked patient categories [Tables 1 and 4].

Frequent abnormal alleles in populations that are likely to select a partner from within their own ethnic group account for virtually all of the remaining homozygous autosomal recessive affected conceptuses. Altogether GeneTests reported 55 diseases that each affects at least 1 in 100,000 patients in specific ethnic populations [Additional file 1: Table S1F]. For the general U.S. population, the first five listed ethnicities each comprise at least $2 \%$ of the total U.S. population and together have 31 ethnic specific diseases that can be added readily to the general population screening test [Additional file 1: Table S1F, \#1-31]. Most ethnic gene mutations require substantially less effort to test because these typically include very few frequent abnormal alleles.

The summary of these individual calculated affected and carrier disease frequencies are listed [Table 4] as well as the composite rates for Caucasian and Worldwide patient categories [Table 1]. These most frequent disease lists were constructed to facilitate physicianselected disease testing that could be ordered for four patient categories: (1) patients selecting a partner or reproducing, (2) at-risk conceptuses, (3) abnormal newborns and minors, and (4) affected adults [Tables 1 and 2].
Currently next generation platforms and their modifications can be used immediately to rapidly sequence the total exome or $\sim 4800$ disease related genetic sites for pregnant couples and affected newborns and adults. Analyzed genes can be selected from among the total results. Fetuses at a 1 in 4 risk of a known gene defect are tested for this risk first. Karyotypes are being ordered initially in Ohio for small invasive prenatal samples, very late gestation fetuses and $90 \%$ of refrigerated POCs that are cultured and karyotyped. Other facilities order microarrays [31] for sufficient CVS and amniocyte samples and up to $40 \%$ of POC samples that can not be cultured. Platforms that complete multiple test categories as well as the most important fetal test will continue to be developed and selected from the most reliable source as test platforms evolve and updated databases are constructed and maintained (Refs. [1,32]; Peer-reviewed publications).

\section{Identifying at-risk couples}

Testing asymptomatic patients prior to or during pregnancy can determine whether both partners carry the same recessive disease gene or the female partner carries an X-linked gene conferring a 1 in 4 risk of each conceptus being affected. Reported population frequencies would identify $40.2 \%$ of Caucasians as carriers of one of the 37 most frequent early onset autosomal recessive diseases [Additional file 1: Table S1A; Table 4.A1.b]. Testing the other current partner will complete the goal of the screening test by identifying the 1 in 174 asymptomatic couples that both carry the same disease allele and have a 1 in 4 risk of an affected conceptus [Table 4 . A.2.b]. Simultaneously the $\sim 1$ in 546 women [Table 4 . B1.b] would be found who carry 1 of 21 most frequent $\mathrm{X}$-linked recessive disease alleles with lower female carrier frequencies [Additional file 1: Table S1B]. Women who test positive for one of these genes have a 1 in 4 risk of an abnormal male fetus so that fetal testing would be offered without testing the partner. Taken together, 1 in $132(.76 \%)$ Caucasian couples are at a 1 in 4 risk for one of the first 61 frequent recessive diseases 
Table 5 Genetic disease loci in critical chromosome regions

\begin{tabular}{|c|c|c|c|c|}
\hline $\begin{array}{l}\text { Chromosome } \\
\text { band tested }\end{array}$ & Gene & Disease locus tested & Disease frequency & OMIM\# \\
\hline \multirow[t]{2}{*}{$1 p 36.3$} & MTHFR & Homocystinuria due to MTHFR & & 236250 \\
\hline & & deficiency & & 607093 \\
\hline \multirow[t]{2}{*}{$1 q 44$} & CIASI & FCAS Muckle-wells syndrome & N.A. & 606416 \\
\hline & & CINCA syndrome & & \\
\hline $2 \mathrm{p} 25$ & TPO & Thyroid peroxidase deficiency & N.A. & 274500 \\
\hline 2q37 N.A. & UGT1A1 & Crigler-Najjar Syndrome, Type II Gilbert Syndrome & N.A & 606785 \\
\hline $3 p 25-p 26$ & $\mathrm{VHL}$ & Von Hippel-Lindau Syndrome & N.A. & 193300 \\
\hline $3 q 27$ or & TP63 & Tumor protein $\mathrm{P} 63$ & N.A. & 603273 \\
\hline $3 q 28$ & LPP & Lipoma-Preferred partner & N.A. & 600700 \\
\hline $4 p 16.3$ or & FGFR3 & Achondroplasia & $1 / 20,000$ & 100800 \\
\hline $4 p 16.3$ & $\mathrm{HD}$ & Huntington Disease & & 143100 \\
\hline $4 \mathrm{p} 35$ & FSHMD1A & Facioscapulohumeral muscular dystrophy & $1 / 250,000$ & 158900 \\
\hline $5 p 15.2-15.3$ & MSR & Methionine Synthase Reductase & N.A. & 602569 \\
\hline $6 \mathrm{p} 25$ or & FOXC1 & Iridogoniodysgenesis & N.A. & 601090 \\
\hline $6 p 25-p 24$ & F13A1 & 13coagulation enzyme & N.A. & 134570 \\
\hline $6 \mathrm{q} 27$ & TBP & Spinocerebellar ataxia 17 & N.A. & 600075 \\
\hline $7 p 22$ & MAD1L1 & Somatic lymphoma & N.A. & 602686 \\
\hline \multirow[t]{2}{*}{$7 q 11.2$} & ELN & Williams Syndrome & $1 / 10,000$ & 194050 \\
\hline & & & & 130160 \\
\hline $7 q 36$ & PRKAG2 & Wolff-Parkinson-White Syndrome & N.A. & 602743 \\
\hline $8 \mathrm{p} 23$ or & $\mathrm{MCPH} 1$ & Microcephaly, autosomal & N.A. & 607117 \\
\hline \multirow[t]{2}{*}{$8 p 22$} & LPL & recessive 1 & $1 / 10,000$ & 238600 \\
\hline & & Hyperlipoproteinemia I & & \\
\hline $8 q 24.3$ & ZIP4 & Acrodermatitis enteropathica & N.A. & 607059 \\
\hline $9 p 24.2$ & PDCD1 & Mouse model develops lupus* & N.A. & 605724 \\
\hline \multirow[t]{2}{*}{$9 q 34.3$} & AGPAT2 & Berardinelli-Seip & N.A. & 603100 \\
\hline & & Congenital Lipodystrophy 1 & & \\
\hline 10p15 & GATA3 & Hypoparathyroidism, sensorineural & N.A. & 131320 \\
\hline $10 q 26$ & AOT & Ornithine Aminotransferase Deficiency & N.A. & 258870 \\
\hline $11 \mathrm{p} 15.5$ & CDKNC1 & Beckwith-Wiedemann Syndrome & N.A. & 600856 \\
\hline $11 q 24$ & KCNJ1 & Bartter Syndrome, Type 2 & N.A. & 600359 \\
\hline $12 \mathrm{p} 13.3$ & WD & Von Willebrand Factor Deficiency & $1 / 20,000$ & 193400 \\
\hline \multirow[t]{2}{*}{$12 q 24.2$} & TCF1 & Diabetes Mellitus & high & 142410 \\
\hline & & Transcription Factor 1 & & \\
\hline $13 q 34$ & IRS2 & Diabetes Mellitus Insulin receptor substrate & & 600797 \\
\hline 14132.33 & IGHM & Agammaglobulinemia & N.A. & 147020 \\
\hline \multirow[t]{2}{*}{$15 q 11.2$} & SNRPN \# & Prader-Willi Syndrome & $1 / 15,000$ & 176270 \\
\hline & UBE3A \# & Angelman Snydrome & $1 / 15,000$ & 601623 \\
\hline $15 q 26.1$ & RECQL3 & Bloom Syndrome & N.A. & 606410 \\
\hline \multirow[t]{2}{*}{$16 p 13.3$} & HBA1 & Alpha Thalassemia & (C) & 141800 \\
\hline & & & & 41850 \\
\hline $16 \mathrm{q} 24.3$ & FANCA & Fanconi Anemia & (D) & 227650 \\
\hline 17p13.3 & LIS1 & Miller-Dieker Syndrome & (E) $90 \%$ deletions & 247200 \\
\hline
\end{tabular}


Table $\mathbf{5}$ Genetic disease loci in critical chromosome regions (Continued)

\begin{tabular}{|c|c|c|c|c|}
\hline \multirow[t]{2}{*}{ 17p11.2 } & \multirow[t]{2}{*}{ PMP22 } & \multirow[t]{2}{*}{ CMT1A/HNPP } & \multicolumn{2}{|l|}{$1 / 5,000(F)$} \\
\hline & & & $20 \%$ de novo & 162500 \\
\hline \multirow[t]{2}{*}{$17 q 25.3$} & \multirow[t]{2}{*}{ HSS } & Sanfilippo Mucopolysaccharidosis & \multirow[t]{2}{*}{ (G) } & 605270 \\
\hline & & Type IIIA & & 252900 \\
\hline $18 p 11.3$ & TGIF & Holoprosencephaly & N.A. & 602630 \\
\hline $18 q 23$ & CYB5 & Methemoglobinemia & N.A. & 250790 \\
\hline 19p13.3 & ELA2 & Cyclic Hematopoiesis & N.A. & 130130 \\
\hline $19 q 13.4$ & TNNT1 & Nemaline myopathy & N.A. & 191041 \\
\hline \multirow[t]{3}{*}{ 20p13 } & \multirow[t]{3}{*}{ AVP } & Diabetes Insipidus & \multirow[t]{3}{*}{ N.A. } & 192340 \\
\hline & & Neurohypophyseal & & 125700 \\
\hline & & Arginine Vasopressin & & \\
\hline \multirow[t]{2}{*}{$21 q 22.3$} & \multirow[t]{2}{*}{ ITGB2 } & \multirow[t]{2}{*}{ Leukocyte adhesion deficiency } & \multirow[t]{2}{*}{ N.A. } & 116920 \\
\hline & & & & 600065 \\
\hline $22 q 11$ & DGCR & DiGeorge Syndrome & N.A. & 188400 \\
\hline \multirow[t]{2}{*}{$22 q 13.3$} & \multirow[t]{2}{*}{ DIA1 } & Methemoglobinemia & \multirow[t]{2}{*}{ N.A. } & \multirow[t]{2}{*}{250800} \\
\hline & & Diaphorase deficiency & & \\
\hline \multirow[t]{3}{*}{ Xp22.32 } & \multirow[t]{3}{*}{ STS } & \multirow[t]{3}{*}{ X-linked ichthyosis } & $1 / 5,000$ & \multirow[t]{3}{*}{308100} \\
\hline & & & Deletions: & \\
\hline & & & $90 \%$ & \\
\hline \multirow[t]{2}{*}{ Xp22.32-pter } & \multirow[t]{2}{*}{ SHOX } & \multirow[t]{2}{*}{ Short Stature Homeo Box } & \multirow[t]{2}{*}{ N.A. } & 604271 \\
\hline & & & & 312865 \\
\hline Xp21.2 & DMD & Duchenne Muscular Dystrophy 65\% deletions, 7 sites, 90\%, 1/3 new mutations & $1 / 4,000$ & 310200 \\
\hline \multirow[t]{2}{*}{ Xq28 } & \multirow[t]{2}{*}{ SLC6A8 } & Creatine deficiency syndrome & & 300352 \\
\hline & & X-linked & & 300036 \\
\hline \multirow[t]{2}{*}{ Yp11.3 } & \multirow[t]{2}{*}{ SRY } & Sex-determining region $Y$ & & \multirow[t]{2}{*}{480000} \\
\hline & & Godndal dysgenesis, XY type & & \\
\hline Yq11.2 & USP9Y & Azoospermia & & 400005 \\
\hline
\end{tabular}

Reproduced from Lebo et al. [30].

[Table 1.1.b, left] and 1/174 Worldwide [Table 1.1.b', right].

Compare targeted testing to the results of screening 23,453 asymptomatic patients that found $29.2 \%$ [24.0\% one allele $+5.2 \%$ more than one allele] Caucasian and ethnic carriers of one of 108 rare and frequent recessive diseases [16]. Testing partners found 1 in 127 couples at a 1 in 4 risk for an affected fetus with 1 of 18 genetic diseases among the 108 tested [16]. Our further analysis found 124 of these 127 at-risk couples in one of two populations: (1) 111 of 127 couples at-risk for 1 of 8 frequent Caucasian diseases (Additional file 1: Table S1A,B); and (2) 13 of 127 couples at-risk for 1 of 7 ethnic diseases in couples with ancestors from the same subpopulation (Table two in Ref. [16]; Additional file 1: Table S1F, This mss.). Only 3 couples were a risk for 1 of the 91 additional less frequent to rare tested diseases [16].

Subsequently our literature study of each of the 34 most frequent worldwide autosomal recessive genetic diseases [Additional file 1: Table S1A] found 28 to 652 reported disease causing mutations [29]. In contrast to cystic fibrosis and the hemoglobinopathies, the 34 worldwide diseases studied had no common mutant alleles so that heterozygous carrier advantage did not select for a few common mutations. Taken together, these comparisons illustrate the efficacy of selecting the most frequent genetic diseases identified and testing for all confirmed disease causing mutations.

Because differences in genetic disease severity or family history may modify the couple's concern about a fetus affected with a reported disease [33], letters a, b, or c have been for more to less severe diseases [Additional file 1: Table S1A, 1-37] Discovering a 1 in 4 risk of an affected fetus for an autosomal recessive disease carried by both partners has modified mate selection. Discovering a 1 in 4 risk of an affected fetus for either an autosomal recessive or X-linked disease enables a couple to select other reproductive options prior to or during pregnancy or to 
optimize care after delivery. When testing each at-risk fetus, simultaneously testing parental samples including any new partners provides optimal controls to further confirm the fetal test result and maintain test accuracy. Reporting only abnormal prenatal results based only on previously reported disease causing genotypes substantially simplifies writing the most accurate reports, counseling, and a couple's decision.

\section{Products of conception and prenatal screening}

Akron Children's product of conception [POC] protocol includes a pathologist's examination, description, biopsy, histology, and submission of freshly biopsied chorionic villi for karyotyping. Chorionic villus biopsies are preferred to other fetal tissues because $90 \%$ of viable submitted fetal samples maintain cell viability when refrigerated up to 5 days prior to cell culture or DNA analysis compared to the poorer viability of other fetal tissues. Histologically prepared villi are analyzed for mole and partial mole morphology (Additional file 4: Table S4). The cytogenetics laboratory assures dissection of the villi to $90-95 \%$ purity for karyotyping and $98-100 \%$ purity for molecular analyses. After digestion and culturing, chorionic villus cells from $90 \%$ of submitted placental samples have been karyotyped. (Additional file 2: Table S2A). Following a preliminary report, polymorphic DNA identity testing confirms the fetal origin of the cultured samples prior to a final report. Of the remaining $10 \%$ of failed cultures, $7 \%$ are sufficiently intact to analyze interphase nuclei by FISH for chromosome 13, 16, 18, 21, 22, X, and Y aneuploidy comprising $\sim 63 \%$ of all chromosome abnormalities in our karyotyped POCs. These $50 \%$ of cultures with abnormal reported karyotypes among all karyotyped POCs are listed in order of frequency for each category [Additional file 2: Table S2A, Col 2]. Given the $90 \%$ of samples karyotyped, these abnormal frequencies are interpreted to reflect typical POC population frequencies. ACOG recommends using microarrays for POC samples when $20 \%-50 \%$ of samples fail to grow in cuture and cannot be karyotyped [34]. Given $90 \%$ of our POCs are karyotyped according to our protocol, we have karyotyped all cultured POCs to identify tetraploidy from diploid genomes and balanced or complex categories of chromosome abnormalities currently missed by microarrays and genomewide sequencing.

Sampled at-risk fetuses have been karyotyped following CVS or amniocentesis to serve as the clinically standard test for microscopically visible chromosome abnormalities. For comparison we added the most recent 12 years of prenatal karyotypes in Ohio to our published 25 years of prenatal karyotypes in San Francisco from 1970 to 1995. This comparison found a 10.6-fold increase in more recent abnormal CVS karyotypes [3.1\% to 33\%] and a 3.2-fold increase in more recent abnormal amniocyte karyotypes (2.3\% to 7.2\%; Ref. [21]; Additional file 2:
Table S2A, Col 3,4) These substantially higher abnormal frequencies in Akron's more recent samples are consistent with those published recently by the American College of Obstetrics and Gynecology [34].

Subsequently these results were organized by severity to emphasize the evolution of surviving abnormal karyotypes as the time after conception increases [Additional file 2: Table S2B)]. These POC frequencies were compared to the reported frequencies in CVS, amniocentesis, and newborn karyotypes (Additional file 2: Table S2B, Col 2,3,4,5; Ref. [21]). Additional categories were observed in POC specimens: (1) triploid to aneuploid,(2) tetraploid to aneuploid, and (3) aneuploid to tetraploid [Additional file 2: Table S2B Col 2, Top, Underlined]. Additional categories were also observed in the more recent amniocyte samples over those previously published: (1) double aneuploidy, (2) isochromosomes, (3) complex abnormalities with two or more abnormal chromosome categories, (4) diploid to tetraploid, (5) trisomy 16 and 22, (6) monosomy 11 and 21, and (7) mosaic karyotypes [Additional file 2: Table S2B, Col 4, Underlined, Italicized].

The 54 selected sites reported to comprise the most frequent chromosome regions involved in abnormal chromosome copy number (Table 5, Reproduced from Ref. [30]; Derived from [32]) were found to have identified all the abnormal chromosome copy number regions identified in the listed abnormal POC, CVS, and amniocyte karyotypes in Akron [Additional file 2: Table S2B]. Because 2,500,000 site polymorphic microarrays only detect abnormal copy number, the frequency of abnormality detection would not have been increased by this assay, but the chromosome region spanned by the copy number change would have been delineated. These abnormal 54 aneuploid loci can be identified on any platform that (1) detects a sufficient number of the most informative adjacent single, di- and tetra-nucleotide polymorphisms and quantifies the relative and total number of times each of these sites were sequenced or (2) the relative number of targets at the aneuploid site compared to a normal diploid control region. Candidate platforms include rapid sequencers and microarrays. Following initial identification of an aneuploid chromosomal gene region on a 54 site test, the extent of the aneuploid gene region could be delineated readily by completing a typical polymorphic genomewide microarray or a $\sim 4800$ disease gene sequence.

Targeting these 54 sites can readily serve as the next generation screening test of circulating placental DNA in maternal circulation [cfDNA] to enable genomewide aneuploid chromosome analysis. These 54 sites comprising the most frequent microscopically visible aneuploid chromosome regions would include sufficiently large genomic targets to compare many polymorphic sites for small differences contributed by fetal DNA. The results 
provided by current and updated placental DNA screening tests are anticipated to continue to be more accurate than prior screening tests. Assuring that reported posterior test accuracy is reported correctly will enable the most confident initial and subsequent screening test utilization.

Alternative platforms and karyotyping should be compared carefully for different applications as tests evolve. For instance, among the $~ 50 \%$ of karyotypically abnormal POC samples, $\sim 45 \%$ of these abnormal samples would have been detected by either a 54 site or a 2,500,000 site genomewide test platform provided the control DNA includes an intact $\mathrm{Y}$ chromosome [Additional file 2: Table S2B, not italicized]. The same platforms would have defined copy number changes in $5.7 \%$ of the $7.2 \%$ [(79\%); (7.2\%-1.54\%)] abnormal amniocyte karyotypes and in 25.7\% of the 33\% [(78\%); (33\%-7.3\%)] current abnormal CVS karyotypes [Additional file 2: Table S2B].

Compare this to microarrays with 2,500,000 sites that precisely map unknown and previously detected unbalanced rearrangements and submicroscopic aneuploidy. These extensive microarrays are particularly useful in delineating the $6 \%$ of inherited cases with undetected submicroscopic deletions or duplications not detected in karyotypes of a conceptus with an apparently balanced chromosomal rearrangement inherited from a normal carrier parent [35]. In spite of the propensity of unequal chromosome recombinations among the potentially confounding $\sim 2,370,000$ copy number variants $[3,36]$, balanced rearrangements are beginning to be detected by improved genomewide platforms and sophisticated computer analysis [17,37]. Karyotyping is the only means to detect diploid to tetraploid mosaicism and tetraploidy arising from diploid cells. As microarray, sequencing, and flow sorting methods for detecting balanced abnormalities are being validated in additional patients with substantial analytic resources,[17] standard karyotyping is still the preferred method for detecting balanced and complex rearrangements in a few viable cells.

Selecting the most optimal platform for any individual fetus will depend upon available ongoing test deveopment and available protocols through referral laboratories. At our location we karyotype all CVS samples because of the 33\% found to have abnormal karyotypes. Currently we karyotype amniocytes for substantially abnormal ultrasounds and select the fetal samples to be sent for microarray analysis following following rapid FISH overnight. Our first sample designated for microarray testing was positive for trisomy 21 by Rapid FISH and subsequent karyotyping.

\section{Symptomatic newborns and children}

Pediatricians following standard of care can decide to test symptomatic newborns and children for all frequent genetic disease categories that may define the abnormal phenotype. These disease categories include the autosomal dominant diseases resulting in $\sim 1$ in 123 affected newborns [Additional file 1: Table S1C, Table 4C] and the frequent deletions and duplications causing an estimated $\sim 1$ in 549 affected newborns [1/1097 X 2; Additional file 3: Table S3A,B, Table 4E]. Together these and all previously mentioned genetic disease categories [Table 1.3] result in $\sim 1$ in 54 [1.86\%] affected newborns worldwide and 1 in 52 [1.91\%] Caucasian newborns.

Calculated dominant disease prevalence reflects a disease frequency that includes many heterozygous abnormal genotypes [2pq] and very few homozygous affected patients [q2] in randomly mating populations with reproducing patients. Mutations involving 60 of these frequent autosomal dominant diseases [Additional file 1: Table S1C, Table 4C] include a substantial proportion that resulted from de novo mutations [2\% to $95 \%$ ]. As anticipated, these de novo autosomal dominant mutations are often more severe than autosomal dominant diseases inherited from reproducing adults. If an autosomal dominant disease gene were identified in an affected minor, parents could then be counseled and offered testing to determine whether the minor's disease gene is de novo [including parental germ line and somatic mosaicism], or resulted from substantial anticipation, variable penetrance, or variable expressivity. Testing these autosomal dominant diseases requires a platform with the sensitivity to detect single nucleotide mutations.

The submicroscopic deletion and duplication category was addressed by analyzing the prior 5 years of abnormal microarray results that each spanned $>400,000$ basepairs in our Texas' patient cohort. These samples were submitted in order of highest to lowest frequency by Neonatologists, Pediatric Neurologists, Geneticists, Developmentalists, and Pediatricians. The 9.6\% abnormal results [121/1265] were organized into three categories [Additional file 3: Table S3B]: (1) 13 loci with frequent deletions with reported normal and abnormal population frequencies that nearly all involved altered neurocognitive development [Additional file 3: Table S3B, Top], (2) at least 23 additional reported loci related to altered neurocognitive development with reported frequencies in abnormal patients [Additional file 3: Table S3B, Middle], and (3) 12 additional clinically significant loci including three chromosomal abnormalities. [Additional file 3: Table S3B, Bottom] About 1/2 of the 123 total deletions and duplications spanned 9 of the first 13 recurrent deletion loci. The other $\sim 1 / 2$ of the cases in categories 2 and 3 included 8 recurrent schizophrenia loci $[24,38]$.

A 52-fold enrichment was found between the 1 in 21 affected patients selected for microarray testing in Texas and an estimated 1 in 1097 patients predicted in the general population by estimated disease frequencies 
[Additional file 3: Table S3B, Top]. This 50-fold enriched frequency of most frequent deletions found among all patients submitted for microarray analysis illustrates the principle that testing clinically suspicious phenotypes substantially enhances the affected patient frequency among tested samples [Additional file 2: Table S2 and Additional file 3: Table S3]. Although a chromosome abnormality was not suspected, 15 cases with trisomy 21, trisomy 18 , or iso (12p) were identified by microarrays in our Texas cohort. Given the difficulty in identifying the exact genetic abnormality by the patient phenotype alone, selected karyotyping may be prudent.

\section{Symptomatic adults}

Symptomatic adults can be tested for all disease categories mentioned previously as well as late onset genetic diseases following appropriate counseling and informed consent. These diseases include Parkinson's disease, Alzheimer disease, and amyotrophic lateral sclerosis in worldwide populations and Huntington disease in Caucasians.

\section{Frequent diseases in specific populations}

The 52 genetic diseases reported to be most frequent in specific populations listed in GeneTests have been incorporated to expedite optimal disease testing in regional laboratories (Additional file 1: Table S1F). Israeli laboratories will want to incorporate platforms with the most frequent Jewish mutations where 6,000,000 Israeli Jews reside, while laboratories in Sweden and Norway would include the Nordic mutations, labs in Quebec the French Canadian mutations, and Asian labs their regionally reported mutations. Worldwide, subpopulations of citizens within specific populations that typically select partners from their own ethnic groups would readily be served by incorporating the few frequent mutations for these diseases into the population wide screening test. These include Asian, Black, and Jewish United States citizens that each comprise at least $2 \%$ of the entire pan ethnic United States population (Additional file 1: Table S1F Group 3; Ref. [39]) and regional laboratories in central Pennsylvania, northeast Ohio, and Colorado where 200,000 Amish and Mennonite regional residents [0.08\% of the total panethnic population] carry $>50$ specific gene mutations reported in these descendents of a few score of founders.

\section{Targeting frequent disease loci enriches clearly defined reportable results}

Currently interpreting genomewide exome sequencing of all $\sim 22,000$ genes requires several times the cost of sequencing. Dr. Hruban reported "a human exome has on average almost 36,000 variants, 45 percent of which are not in an SNP database and about 100 of which can cause loss of function" [40]. He concluded, "The potential power of next-generation sequencing for clinical testing is substantial. It will be a while before it is brought fully to the clinic..." This agrees with Chun et al. who identified $\sim 90$ potential disease causing sequences identified by exome analysis of all $\sim 22,000$ genes in three individuals [5]. When testing $100,000 \mathrm{pa}-$ tients for the frequent autosomal recessive cystic fibrosis mutations with $99.9 \%$ test accuracy per gene, a positive carrier test would include 3445 correct answers and 103 incorrect answers (Table 3, Top; Ref. [20]). In contrast, for the rare fumarase deficiency gene locus with the same test accuracy a positive carrier test would not only include 26 correctly analyzed carriers but also 100 incorrect false positive carrier test results [Table 3, Bottom]. Maintaining the principle of testing the most frequent diseases listed from most to less frequent in each selected category provides an objective basis to select the lowest frequency of any tested disease in each category.

\section{Discussion}

In summary. analyzing the 227 diseases affecting 263 loci that are each reported to affect at least 1 in 100,000 Caucasian individuals together include $\sim 1.2 \%$ of all the $22,000$ gene loci [ 1 in 84$]$, and $~ 9.1 \%$ [ 1 in 11] of the $>2500$ listed clinically testable disease causing genes [1]. Based upon these frequencies, analyzing all $>2500$ known disease causing genes is estimated to identify $\sim 11$ potential disease causing genes. In contrast, testing the attached list of most frequent 227 disease loci in the United States [Additional file 1: Table S1, Additional file 2: Table S2 and Additional file 3: Table S3] is anticipated to decrease the number of candidate affected genotypes to be considered to $\sim 0-2$ per patient. This list of frequent diseases includes $\sim 7$ of 8 of McKusick's (1982) estimated single locus Caucasian birth defects $\{[1.91 /(0.4 \%+1.8 \%)=87 \%]$ Table 1.3a, Ref. [41] $\}$ and $\sim 6$ of 7 Worldwide birth defects without region specific testing. [Derived from McKusick's estimated 1.8\% transmitted by Mendelian inheritance plus $0.4 \%$ chromosome abnormalities]. These most frequent diseases affect about 1 in 52 Caucasian newborns and 1 in 54 worldwide [Table 1.3.a,a'] These listed sites [Additional file 1: Table S1, Additional file 2: Table S2 and Additional file 3: Table S3] focus testing to the fewest most informative disease gene locations to achieve the highest possible test accuracy (Table 3; Refs. $[20,42])$. Because abnormal phenotypes are frequently seen in more than one genetic disease or chromosome abnormality, this targeted genomewide patient category testing approach can readily identify suspected and unsuspected abnormalities too numerous to test individually.

The 54 targeted chromosomal sites that would have identified all the microscopically visible aneuploid chromosome regions in our reported karyotypes reflect the efficacy of targeting the most frequent disease 
causing rearrangements. Currently microarrays and exome sequencing identify nearly all Genomewide normal and abnormal modifications. Genomewide platforms and computer programs can now be targeted to analyze only the most frequent disease causing sites [Additional file 1: Table S1, Additional file 2: Table S2 and Additional file 3: Table S3]. Platforms and computer programs that reveal physician selected patient categories and the diseases to be included will further enhance laboratory turnaround time [Figure 1A,B,C; Table 1]. Any selected platform category is likely to determine copy number. Upon further modification, rapid sequencing platforms and/or polymorphic or sequencing microarrays are anticipated to enable analyzing the most frequent reported disease causing mutations at fewer tested sites to most efficiently utilize all manufacturing, testing, interpretation, and counseling resources. Reporting only peer reviewed published mutations with their references would enable actionable results while minimizing inconclusive test risks.

The 13 frequent deletions reported in $\sim 1$ in 21 of the patients tested by microarrays were $\sim 52$-fold more frequent in this physician selected population than in the general population [1 in 1097; Additional file 3: Table S3B, Top)]. Although the relative contribution of each submitted patient category will be modified according to the physician's specialty, substantial enrichment of abnormal genotypes is anticipated in all affected patient categories submitted for testing. This will improve upon total test accuracy.

Given the use and analysis of large genome data in clouds, companies are offering sufficient computing capability to analyze and store genomewide sequencing data for subsequent reanalysis. Given multiple reanalyses could be applied to the same data set as additional disease genes are discovered, disease gene mutations are cataloged, and new clinical information becomes available, stored genomewide data can be reanalyzed without resequencing. Laboratories that choose to provide this service can write additional programs to analyze all the data and distribute summaries of new findings to contributing physicians. All this requires prior patient understanding and agreement to receive updated information by continuing these analyses. The Perspective by Dr. Pyeritz addresses possible legal implications of reporting or of not reporting newly discovered genes [43]. Ongoing modifications to maintain optimal test platforms can be based upon additional identified disease genes, a change in individual observed disease frequency in sampled affected patients, ongoing test results, the geographical origin(s) of tested patients, and additional published causative mutations.

The U.S. Army Corps of Engineers Motto is, "The difficult we do immediately. The impossible takes a little longer". Computer programs that reveal only physician selected gene results on the core panel can immediately optimize patient specific testing and minimize laboratory liability for unreported loci. Targeting and testing the most frequent genetic abnormalities on a single platform [Additional file 1: Table S1 A-G] will identify most clinically meaningful abnormal genotypes for any designated patient category. Testing all the confirmed disease associated genes with the most frequent disease core would provide $\sim 10$-fold less data than the total disease causing exome platform. The following applications can be considered immediately:

1. Testing the 54 most frequent chromosomal sites to identify most microscopically visible karyotypic abnormalities in fetal DNA in maternal circulation.

2. Targeting less than 64 frequent worldwide genomic abnormalities in the core list [Additional file 1: Table S1A,B] to readily identify the largest proportion of couples at-risk for affected fetuses worldwide.

3. Targeting $\sim 257$ listed genomic sites (Additional file 1: Table S1A-E) would identify most known genetic disease-causing mutations in abnormal children.

4. Developing targeted genomewide testing for the most frequent abnormalities including both single nucleotide mutations and gene aneuploidy on a single platform (Additional file 1: Table S1, Additional file 2: Table S2 and Additional file 3: Table S3) to optimize genomewide testing.

5. Computer programs written to only reveal each physician selected patient category and genes within it on any genomewide panel enables targeted testing with the fewest platforms.

6. Adding population-specific frequent disease mutations according to a testing laboratory's geographical location.

\section{Conclusion}

This principle of selecting and testing the most frequent genomewide disease causing abnormalities in $\sim 1$ of 8 known disease loci ( 1 of 84 total gene loci) is estimated to identify the genetic defect in $\sim 7$ of 8 reported abnormal newborn Caucasians. In contrast, this would eliminate $\sim 8$ to10 of $\sim 10$ Caucasian newborn gene sequences selected as abnormal that are actually normal variants identified when testing all $\sim 4800$ reported disease genes to search for the remaining 1 of 8 disease causing genes. Adopting this approach will minimize incorrect results while optimizing test accuracy, counseling, and reimbursement for a larger proportion of appropriate patients within available laboratory and reimbursement resources. 


\section{Additional files}

\section{Additional file 1: Table S1. Most frequent genetic diseases by} transmission category [25,44-47].

Additional file 2: Table S2A. 26 Abnormal karyotypic categories [48] detected at 54 sites (Table 5) P27-29 [31]. Listed by frequency in POCs. Table S2B. Abnormal karyotypic categories detected at 54 sites. Listed by decreasing severity.

Additional file 3: Table S3. Common submicroscopic aneuploid locl by chromosome location and category: $[24,48]$.

Additional file 4: Table S4. Current platform detection capability [49-56].

\section{Competing interests}

Dr. Lebo has an issued patent that teaches analysis of the most frequent genetic diseases optimizes genomewide analysis: Lebo RV, Wyandt HE, Milunsky A. Optimizing Genome-Wide Mutation Analysis of Chromosomes and Genes. U.S. Patent 8,548,747 [30]. Owned by Akron Children's Hospital. Dr. Tonk has no competing interest.

\section{Authors' contributions}

VST contributed his laboratory's microarray results for which he completed analysis and reported clinically. VST's laboratory also provided his clinically reported Products of Conception karyotypes. The Akron Children's prenatal dataset is presented. RVL conceived the study, selected and derived the disease frequency tables and statistical analysis, and wrote the manuscript. Both authors read and approved the final manuscript's content.

\section{Acknowledgments}

We thank Robert Novak, M.D., Chairman Akron Children's Pathology Department, for ongoing insightful commentary. For support and assistance we thank Dr. Richard Lampe, Chairman, and John Berry, Department of Pediatrics, Texas Tech University Health Science Center (TTUHSC), Catherine Lovett and Dr. L.O. Luther at TTUHSC Clinical Research Institute, and TTUHSC Genetics Division employees and referring physicians in house and throughout West Texas including Lubbock, Odessa, El Paso and Amarillo. We thank Cortney Becker for TTUHSC microarray and POC compilation. We also thank Thaddeus Kurczynsky, M.D., Ph.D., FACMG and Haynes Robinson, M.D. FACMG at Akron Children's for commentary, Dr. James Saller for review, and Franklin Barr Lebo, J.D., Ph.D., for editorial assistance.

\section{Author details}

'Department of Pathology and Laboratory Medicine, Akron Children's Hospital, One Perkins Square, Akron, OH 44308-1062, USA. ${ }^{2}$ Northeast Ohio Medical University, Rootstown, OH, USA. ${ }^{3}$ Department of Pediatrics, Texas Tech University Health Science Center, 4th Street 3601, Lubbock, TX 79416, USA. ${ }^{4}$ Texas Tech University Health Sciences Center, El Paso, TX, USA.

\section{Received: 12 April 2014 Accepted: 14 November 2014}

Published online: 21 January 2015

\section{References}

1. GeneTests/GeneReviews in NIH Genetic Testing Registry (GTR). [http://www. genetests.org/by-genereview/] [http://www.ncbi.nlm.nih.gov/sites/ GeneTests/]

2. Affymetrix, Genome-Wide Human SNP Array 6.0. [06.15.14.]. [http://www. affymetrix.com/ estore/catalog/131533/AFFY/Genome-Wide-Human-SNPArray-6.0\#1_1,

3. Toronto Database of Genomic Variants. [http://dgv.tcag.ca/dgv/app/ statistics?ref=NCBI36/hg18]

4. Clark W. Finding a clinical fit for next-gen sequencing. CAP Today Coll Am Pathol. 2012;26:1-48.

5. Chun S, Fay JC. Identification of deleterious mutations within three human genomes. Genome Res. 2009;19:1553-61.

6. Watson J. Invited Panelist. Montreal, CA: International Congress of Human Genetics; 2011. http://www.rickilewis.com/blog.htm?post=817161.

7. Kohane IS, Hsing M, Kong SW. Taxonomizing, sizing, and overcoming the incidentalome. Genet Med. 2012;14:399-404.
8. Grody WW, Cutting GR, Klinger KW, Richards CS, Watson MS, Desnick RJ, et al. Laboratory standards and guidelines for population-based cystic fibrosis carrier screening. Genet Med. 2001;3:149-54.

9. ACOG, The American College of Obstetricians and Gynecologists Committee Opinion No. 486. Update on Carrier Screening for Cystic Fibrosis. Obstet Gynecol. 2010;117:1028-31 [http://www.acog.org/ /media/ Committee\%200pinions/Committee\%20on\%20Genetics/co486.pdf? $\mathrm{dmc}=1 \& \mathrm{ts}=20140707 \mathrm{~T} 1518236472]$

10. Lebo RV. Testing and reporting ACMG cystic fibrosis mutation panel results. [abstract]. Genet Test. 2007;11:11-31.

11. Lebo RV, Grody WW. Variable penetrance and expressivity of the splice altering 5T sequence in the cystic fibrosis gene [abstract]. Genet Test. 2007;11:32-44.

12. Monaghan KG, Feldman GL, Palomaki GE, Spector EB, Ashkenazi Jewish Reproductive Screening Working Group, the Molecular Subcommittee of the ACMG Laboratory Quality Assurance Committee. Technical standards and guidelines for reproductive screening in the Ashkenazi Jewish population. Genet Med. 2008;10:57-72.

13. Dor Yeshorim-Modern Diagnostic Laboratory Inc. [http://modernlab.org/doryeshirum.html]

14. Caskey $C T$. Using genetic diagnosis to determine individual therapeutic utility. Ann Rev Med. 2010:61:1-15.

15. Caskey CT, Gonzalez-Garay ML. Genetic Diagnostics using Next-Generation Sequencing: The CEO Genome Project [Abstract]. Toronto: International Cong. Human Genetics; 2011. Abstract \#s46.

16. Lazarin GA, Haque IS, Nazareth S, Lori K, Patterson AS, Jacobson JL, et al. An empirical estimate of carrier frequencies for $400+$ Caucasian Mendelian variants: Results from an ethnically diverse clinical sample of 23,453 individuals. Genet Med. 2012;14:1-9.

17. Jensen TJ, Kim SK, van den Boom D, Deciu C, Ehrich M: Noninvasive Detection of a Balanced Fetal Translocation from Maternal Plasma. Clin Chem, 2014. http://www.clinchem.org/content/early/2014/07/15/clinchem.2014.223198. short?rss=1.

18. TessArae [http://www.tessarae.com/downloads/Manuals/ TessArray_PECSP_Manual.pdf]

19. Illumina [http://wwwillumina.com/]

20. Lebo RV, Grody WW: Standard-of-care screening beyond cystic fibrosis: Targeting the most frequent genetic abnormalities [abstract]. Am Soc Hum Genet, 2010. Platform Presentation. [http://www.ashg.org/2010meeting/ abstracts/fulltext/f21240.htm]

21. Lebo RV, Flandermeyer RR, Lynch ED, Lepercq JA, Diukman R, Golbus M. Prenatal diagnosis with repetitive in situ hybridization probes. Am J Med Genet. 1992:43:848-54

22. BlueGnome/llumina [http://www.cambridgebluegnome.com/about]

23. Agilent Home [www.home.agilent.com/agilent/home.jspx?cc=US\&lc=eng]

24. Kaminsky EB, Kaul V, Paschall J, Church DM, Bunke B, Kunig D, et al. An evidence-based approach to establish the functional and clinical significance of copy number variants in intellectual and developmental disabilities. Genet Med. 2011;13:777-84.

25. Vogel F, Motulsky AG. Human Genetics. 2nd ed. Berlin: Springer Verlag 1986. p. 335

26. Luzzatto L, Nwachuku-Jarrett ES, Reddy S. Increased sickling of parasitised erythrocytes as mechanism of resistance against malaria in the sickle-cell trait. Lancet. 1970:1(7642):319-21.

27. Friedman MJ. Erythrocytic mechanism of sickle cell resistance to malaria. Proc Natl Acad Sci U S A. 1978;75:1994-7.

28. Fleming AF, Storey J, Molineaux L, Iroko EA, Attai ED. Abnormal haemoglobins in the Sudan savanna of Nigeria. I. Prevalence of haemoglobins and relationships between sickle cell trait, malaria and survival. Ann Trop Med Parasitol. 1979;73:161-72.

29. Gener MAH, Lebo RV. Most autosomal recessive diseases have no frequent mutations that reflect increased carrier fitness or mutation hotspots. American Society Human Genetics, October 22-26, Boston, MA. Poster 2014F, 2013.

30. Lebo RV, Wyandt HE, Milunsky A: Optimizing Genome-Wide Mutation Analysis of Chromosomes and Genes. U.S. Patent 8,548,747. Owned by Akron Children's Hospital, October 1, 2013. http://patft.uspto.gov/netacgi/nph-Parser?Sect1=PTO2\&Sect2=HITOFF\&u=\%2Fnetahtm|\%2FPTO\%2Fsearch-adv. $h t m \& r=1 \& f=G \& l=50 \& d=P T X T \& p=1 \& S 1=8,548,747 \& O S=8,548,747 \& R S=8,548,747$.

31. Bug S, Solfrank B, Schmitz F, Pricelius J, Stecher M, Craig A, et al. Diagnostic utility of novel combined arrays for genome-wide simultaneous detection of aneuploidy and uniparental isodisomy in losses of pregnancy. Mol Cytogen. 2014;7:43-52. 
32. Online Mendelian Inheritance in Man (OMIM): [http://www.ncbi.nlm.nih.gov/ omim?TabCmd=Limits]

33. Grody WW, Thompson BH, Gregg AR, Bean LH, Monaghan KG, Schneider A, et al. ACMG position statement on prenatal/preconception expanded carrier screening. Genet Med. 2013;15:482-3.

34. ACOG, The American Congress of Obstetricians and Gynecologists Committee on Genetics Society for Maternal-Fetal Medicine Committee Opinion No. 581: The Use of Chromosomal Microarray Analysis in Prenatal Diagnosis. 2013. [http://www.acog.org/Resources_And_Publications/Committee_ Opinions/Committee_on_Genetics/

The_Use_of_Chromosomal_Microarray_Analysis_in_Prenatal_Diagnosis]

35. Sismani C, Kitsiou-Tzeli S, loannides M, Christodoulou C, Anastasiadou V, Stylianidou G, et al. Cryptic genomic imbalances in patients with de novo or familial apparently balanced translocations and abnormal phenotype. Mol Cytogenet. 2008;1:15-24.

36. Kiyosawa $\mathrm{H}$, Chance PF. Primate origin of the CMT1A-REP repeat and analysis of a putative transposon-associated recombinational hotspot. Hum Mol Genet. 1996;5:745-53.

37. Duncavage EJ, Abel HJ, Szankasi P, Kelley TW, Pfeifer JD. Targeted next generation sequencing of clinically significant gene mutations and translocations in leukemia. Mod Pathol. 2012;25:795-804.

38. Miller DT, Adam MP, Aradhya S, Biesecker LG, Brothman AR, Carter NP, et al. Consensus statement: chromosomal microarray is a first-tier clinical diagnostic test for individuals with developmental disabilities or congenital anomalies. Am J Hum Genet. 2010;86:749-64.

39. Jewish Population by State [C:IContent.Outlook12Z1Z0GCNewish Population of the United States by State.mht]

40. Hruban, quoted in Clark, W: Finding a clinical fit for next-gen sequencing. CapToday 2012, 26:1-48 [http://digital.olivesoftware.com/Olive/ODE/CapToday/PrintComponentView.htm]

41. McKusick V. Presentation to HHMI Genetics Investigators Conference Howard Hughes Medical Institute: Hialeah, FL; 1982.

42. Lebo RV, Grody WW: Genomewide Testing the Most Frequent Genetic Diseases Optimizes Abnormal Gene Identity and Test Accuracy [abstract]. Montreal, Canada: International Congress of Human Genetics; 2011. Platform Presentation, First Submitted Abstract Secession. Abstract \#44. [http://www. ichg2011.org/abstracts/fulltext/f20402.htm]

43. Pyeritz RE. The coming explosion in genetic testing-Is there a duty to recontact? New Engl J Med. 2011:365:1367-9.

44. De Grouchy J, Turleau C. Clinical Atlas of Human Chromosomes. 2nd ed. New York: John Wiley \& Sons; 1984

45. Thompson MW, Mclnnes RR, Willard HF. Thompson and Thompson: Genetics in Medicine. 5th ed. Philadelphia: W.B. Saunders Co., Harcourt, Brace, Jovanovich, Inc.: 1991.

46. Nussbaum RL, McInnes RR, Willard HF. Genetics in Medicine. 7th ed. Philadelphia: Saunders/Elsevier; 2007.

47. Jorde LB, Carey JC, Bamshad MJ, White RL. Medical Genetics. 3rd ed. St. Louis: Mosby; 2003. p. 98

48. Applied. Not found in database.-RVL48. Lebo RV, Tonk VS: Analyzing the Most Frequent Disease Loci in Targeted Patient Categories Optimizes Disease Gene Identification and Test Accuracy Worldwide. Nashville, TN: American College of Medical Genetics Conference; 2014. Abstract \# 270. [http://ww2.aievolution.com/acm1401/index.cfm?do=abs. viewAbs\&abs=2452]

49. Kajii T, Niikawa N. Origin of triploidy and tetraploidy in man: 11 cases with chromosome markers. Cytogenet Cell Genet. 1977;18:109-25. Tetraploidy.

50. Meulenbroek GHM, Geraedts JPM. Parental origin of chromosome abnormalities in spontaneous abortions. Hum Genet. 1982;62:129-33.

51. Sheppard DM, Fisher RA, Lawler SD, Povey S. Tetraploid conceptus with three paternal contributions. Hum Genet. 1982;62:371-4.

52. Slim R, Mehio A. The genetics of hydatidiform moles: new lights on an ancient disease Clin Genet. 2007:71:25-34

53. Fukunaga M. Immunohistochemical characterization of p57(KIP2) expression in early hydatidiform moles. Hum Pathol. 2002;33:1188-92.

54. Fukunaga M. Immunohistochemical characterization of p57 expression in tetraploid hydropic placentas. Archives Pathol Lab Med. 2004;128:897-900.

55. Lau CC, Davis C, Rao P, Selzer R, Eis P: Detection of balanced translocation by DNA microarrays. http://www.ashg.org/genetics/ashg07s/f21248.htm.

56. Vejerslev LO, Dissing J, Hansen HE, Poulsen H. Hydatidiform mole: genetic origin in polyploid conceptuses. Hum Genet. 1987:76:11-9.

\section{Submit your next manuscript to BioMed Central and take full advantage of:}

- Convenient online submission

- Thorough peer review

- No space constraints or color figure charges

- Immediate publication on acceptance

- Inclusion in PubMed, CAS, Scopus and Google Scholar

- Research which is freely available for redistribution

Submit your manuscript at www.biomedcentral.com/submit 